\title{
Evaluation of Ventricular Polarization in Noncompaction Cardiomyopathy with Electrocardiography in a Different Perspective
}

\section{(1) Onur Akhan, (1) İsa Ardahanlı}

Bilecik State Hospital, Clinic of Cardiology, Bilecik, Turkey

\begin{abstract}
Objectives: Noncompaction cardiomyopathy (NCCM) is a rare genetic form of cardiomyopathy. Morphologically, it consists of a thickened two-layer myocardial structure (noncompacted-compacted layers.). In NCCM, especially in highly symptomatic cases, abnormal basal electrocardiographic (ECG) patterns can be observed at all stages of atrial and/or ventricular depolarization and repolarization. In recent studies, it has been shown that increased QT duration, fragmented QRS pattern, increased spatial QRS-T angle could be predictive concerning ventricular arrhythmias in NCCM. We aimed to show the relationship (if any) between the parameters related to ventricular depolarization and repolarization, which are QT variability/dispersion (QTd), TPe, TPe/QT ratio, "the index of Cardiac-Electrophysiologic Balance (iCEB)" and NCCM.
\end{abstract}

Materials and Methods: Thirty NCCM patients and 31 healthy volunteers were included in this cross-sectional, casecontrol study. ECG parameters related to ventricular polarization were evaluated for all participants.

Results: In our study, we observed that TPe, corrected TPe (cTPe), TPe/QT, TPe/corrected QT (cQT) and cTPe/cQT values were significantly lower in NCCM patients compared to controls (all p values $<0.005$ ). QTd and corrected QTd (cQTd) values are also relatively low in NCCM ( $p>0.05)$.

Conclusion: We found a significant relationship between NCCM and TPe, cTPe, TPe/QT, TPe/cQT, cTPe/cQT parameters. It can be thought that our study contributed to the literature with the present findings and it may also be a guide for clinical trials to investigate the relationship of these parameters with ventricular arrhythmias in NCCM.

Keywords: Arrhythmia, electrocardiography, cardiomyopathy

Address for Correspondence: Onur Akhan, Bilecik State Hospital, Clinic of Cardiology, Bilecik, Turkey

e-mail: akhanonur@gmail.com ORCID: orcid.org/0000-0003-4440-9599

Received: 31.08 .2020 Accepted: 13.11 .2020

Cite this article as: Akhan O, Ardahanlı İ. Evaluation of Ventricular Polarization in Noncompaction Cardiomyopathy with

Electrocardiography in a Different Perspective. EJCM 2020;8(4):180-185.

DOI: 10.32596/ejcm.galenos.2020.08.044

${ }^{\circ}$ Copyright 2020 by Heart and Health Foundation of Turkey (TÜSAV) / E Journal of Cardiovascular Medicine published by Galenos Publishing House. 


\section{Introduction}

Noncompaction

cardiomyopathy

(NCCM)

morphologically consists of increased trabeculation and a thickened two-layer myocardial structure ${ }^{(1,2)}$. Congestive heart failure, arrhythmias, thromboembolic events can be seen in $\mathrm{NCCM}^{(3)}$. However, in recent years, asymptomatic cases have increased with the increase of screening ${ }^{(4)}$. Especially in highly symptomatic cases, abnormal electrocardiogram (ECG) patterns such as atrial fibrillation, atrioventricular block, bundle branch blocks, and Wolff-Parkinson-White pattern were observed. ECG abnormalities can be observed at all stages of atrial and/or ventricular depolarization and repolarization ${ }^{(5,6)}$. Findings of left ventricular hypertrophy (LVH), PR and QT prolongation, and $\mathrm{T}$ wave changes can be counted among other ECG changes ${ }^{(7)}$. Beta-blocker therapy is considered as a safe treatment for the suppression of arrhythmias, especially in the group with heart failure. Class 3 antiarrhythmic drugs can also be used in appropriate conditions ${ }^{(8)}$. Implantable cardioverterdefibrillator (ICD) and cardiac resynchronization therapy (CRT) treatment modalities can also be applied within the clinical indications in the follow-up of the patients ${ }^{(9)}$.

In recent studies, it has been shown that increased QT duration, fragmented QRS (fQRS) pattern, increased spatial QRS-T angle could be predictive concerning ventricular arrhythmias, morbidity and mortality in $\mathrm{NCCM}^{(10,11)}$.

In studies conducted related to ventricular polarization abnormalities and arrhythmic events, it was stated that the QTd and the TPe could be used as a biomarker for torsades de pointes risk assessment ${ }^{(12-14)}$. Similarly, the TPe/QT ratio can be used as a biomarker because it gives information about the transmural dispersion of LV repolarization ${ }^{(15-17)}$. In 2016, Chua et al. ${ }^{(18)}$ examined the ECG of patients who had a history of sudden cardiac arrest (SCA) and showed that TPe value could be an independent predictor of SCA. It was also stated that the values of QT and TPe should be corrected according to the heart rate. As a result of the measurements made using the Bazett formula, it was concluded that the cTPe above $90 \mathrm{~ms}$ increased the risk almost three times. Based on electrophysiological studies, in 2013, it has been revealed that the QT/QRS ratio can be used as a new biomarker to detect cardiac arrhythmias. Such an inference has been made because changes in the QT time are proportional to changes in the effective refractory period (ERP) time and changes in the QRS wave duration are proportional to conduction velocity. This biomarker is named as "iCEB"(19).

Based on these studies and with the plan to look at the parameters that have not been studied before at about NCCM, in our article, it is aimed to show the relationship (if any) between NCCM and the parameters related to ventricular depolarization and repolarization which are QTd, TPe, TPe/QT ratio and iCEB.

\section{Materials and Methods}

Our study is a cross-sectional, case-control study. Thirty NCCM patients (18-65 ages) who were diagnosed through cardiac magnetic resonance (CMR) (using Peterson Criteria) were included ${ }^{(20)}$. Ischemic cardiomyopathy, peripartum cardiomyopathy, chronic alcohol use, chemotherapeutic drug use with known cardiotoxic effect, body mass index $>35 \mathrm{~kg} /$ $\mathrm{m}^{2}$, infiltrative diseases, endocrine disorders, acute/ chronic kidney disease, myocarditis, moderate/severe heart valve diseases, cardiac intervention/operation (pacemaker, ICD, CRT implantation, left ventricular assist device, heart transplantation) history, and reversible situations which could cause left ventricular noncompaction were determined as exclusion criteria. All the patients were in sinus rhythm; LVH, cardiac blocks, antiarrhythmic drug usage other than betablockers, antihistamine, tricyclic antidepressants, and antipsychotics usage were also excluded. Beta-blocker usage could not be excluded due to the treatment being included in the optimal medical treatment for heart failure. The control group consisted of age- and sexmatched 31 healthy volunteers. Informed consent was obtained from all patients and local ethics committee 
approval was received (Ege University Ethics Committee, with date: 8/8/17, number: 17-6/12).

Standard echocardiographic measurements were made in reference with 2015 ASE (American Society of Echocardiography) guideline ${ }^{(21)}$. $\mathrm{NC} / \mathrm{C}$ ratio was calculated by echocardiography at the end of systole with the Jenni criterion ${ }^{(22)}$. Parameters related to CMR were obtained from patients' hospital reports.

\section{Electrocardiographic Evaluation}

The standard 12-lead surface ECGs of patients were obtained at rest in the supine position $(10 \mathrm{mV} / \mathrm{mm}$ and 50 $\mathrm{mm} / \mathrm{s}$ paper speed; with recorder Nihon Kohden, Japan). After scanning, all ECGs were analyzed in a digital platform with $\mathrm{x} 400$ magnification to diminish the error. Measurements of ECGs were performed by two separate cardiologists. Heart rate and QRS duration data were taken from the automated ECG report. The QT interval was calculated from the onset of the QRS complex to the end of the $T$ wave in as many leads as possible (minimum and maximum values of QT were also recorded.). TPe interval was calculated from the peak point to the end of $\mathrm{T}$ wave in $\mathrm{V} 5$, followed by other precordial leads if V5 was not appropriate (Figure 1). The QT and TPe

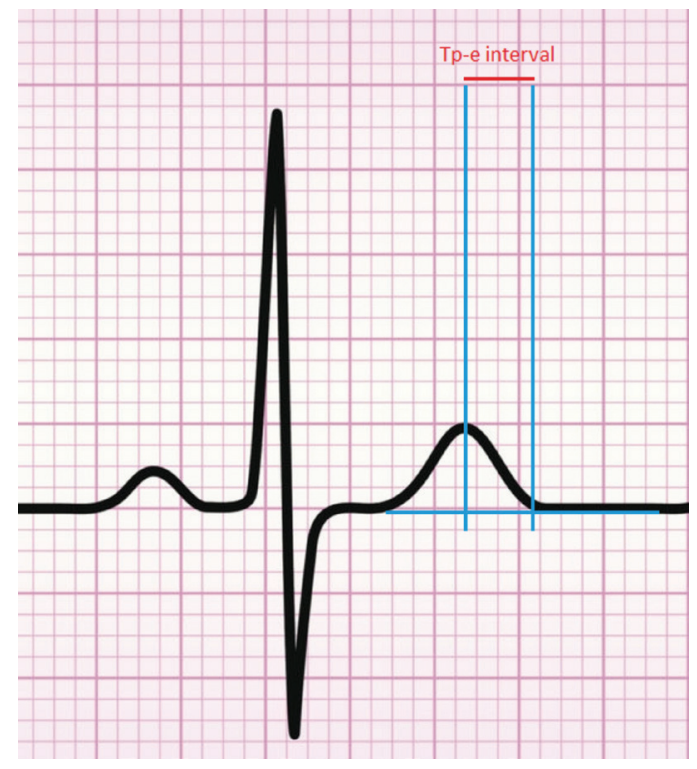

Figure 1. Measurement of TP-e interval TP-e: T-peak to T-end intervals were corrected for heart rate using the Bazett formula (QT/ $\left.\sqrt{ } \mathrm{RR}-\mathrm{TPe} /{ }^{3} \sqrt{ } \mathrm{RR}\right)^{(18)}$. QTd was calculated as the difference of $\mathrm{QT}$ maximum $\left(\mathrm{QT}_{\max }\right)$ and minimum $\left(\mathrm{QT}_{\min }\right)$ values and corrected QTd (cQTd) was calculated also as the difference in corrected QT maximum ( $\left.\mathrm{cQT}_{\text {max }}\right)$ and corrected QT minimum ( $\mathrm{cQT}_{\text {min }}$ ). TPe/QT $\mathrm{T}_{\max }, \mathrm{cTPe} /$ $\mathrm{cQT}_{\max }, \mathrm{QT}_{\max } / \mathrm{QRS}$ (also named as $\mathrm{iCEB}$ ), $\mathrm{cQT}_{\max } / \mathrm{QRS}$ (also named as iCEBc) were other measured parameters ${ }^{(19)}$. iCEB was calculated by the formula using $\mathrm{QT}_{\max }, \mathrm{cQT}_{\max }$, and QRS duration values in our data. Subjects with U waves and ECGs that were not suitable for evaluation were excluded.

\section{Statistical Analysis}

Statistical analysis was carried out by using SPSS software (version 22.0, SPSS Inc., Chicago, IL, US). Continuous variables were expressed as median while categorical variables were shown as a percentage (\%). The Student t-test or Mann-Whitney U test were used for continuous variables, and Fisher's exact test or chi-square test, whichever was suitable, was used for categorical variables. Values with $\mathrm{p}<0.05$ were considered statistically significant.

\section{Results}

Basal characteristics of the study population are summarized in Table 1. There were no significant differences between the groups regarding age, gender and body surface area (BSA). In the patient group, two patient had diabetes mellitus (DM) and nine patients had hypertension (HT). Beta blocker usage rate was $\% 78.1$ for the patients (The beta blocker agents used by patients were carvedilol and metoprolol. The maximum doses were 25 $\mathrm{mg}$ twice a day for carvedilol and $100 \mathrm{mg}$ once a day for metoprolol.).

In the patient group, the mean value of left ventricular ejection fraction was 43.4 , and significantly lower than in the control group $(\mathrm{p}<0.001)$. Left atrium, left ventricular end-diastolic and end-systolic diameters (LVEDDLVESD) were higher in the patient group ( $<<0.05$, only for LVEDD $\mathrm{p}=0.166$ ). The mean heart rates were similar 
( $p=0.989$ ) but the $\mathrm{QRS}$ duration was significantly higher in the patient group ( $\mathrm{p}=0.006)$. Other ECG findings are showed in Table 2.

Although the mean $\mathrm{QT}_{\max }, \mathrm{QT}_{\text {min }}, \mathrm{QTd}$, and also their corrected measurements (cQT $\mathrm{cmax}_{\text {man }}, \mathrm{cQT}_{\min }, \mathrm{cQTd}$ ) were higher and $\mathrm{iCEB}$ and $\mathrm{iCEBc}$ were lower in the patient group compared to the control group, no significant difference was observed $(\mathrm{p}>0.05)$. TPe and TPe related parameters, which were cTPe, TPe/QT ratio, TPe/cQT ratio, and cTPe/ cQT ratio, were observed to be significantly higher in the patient group compared to the control group (all $\mathrm{p}$ values $<0.05)$.

\section{Discussion}

In the studies carried out to date to determine the electrocardiographic features of the NCCM and the risk

Table 1. Characteristics of the study population

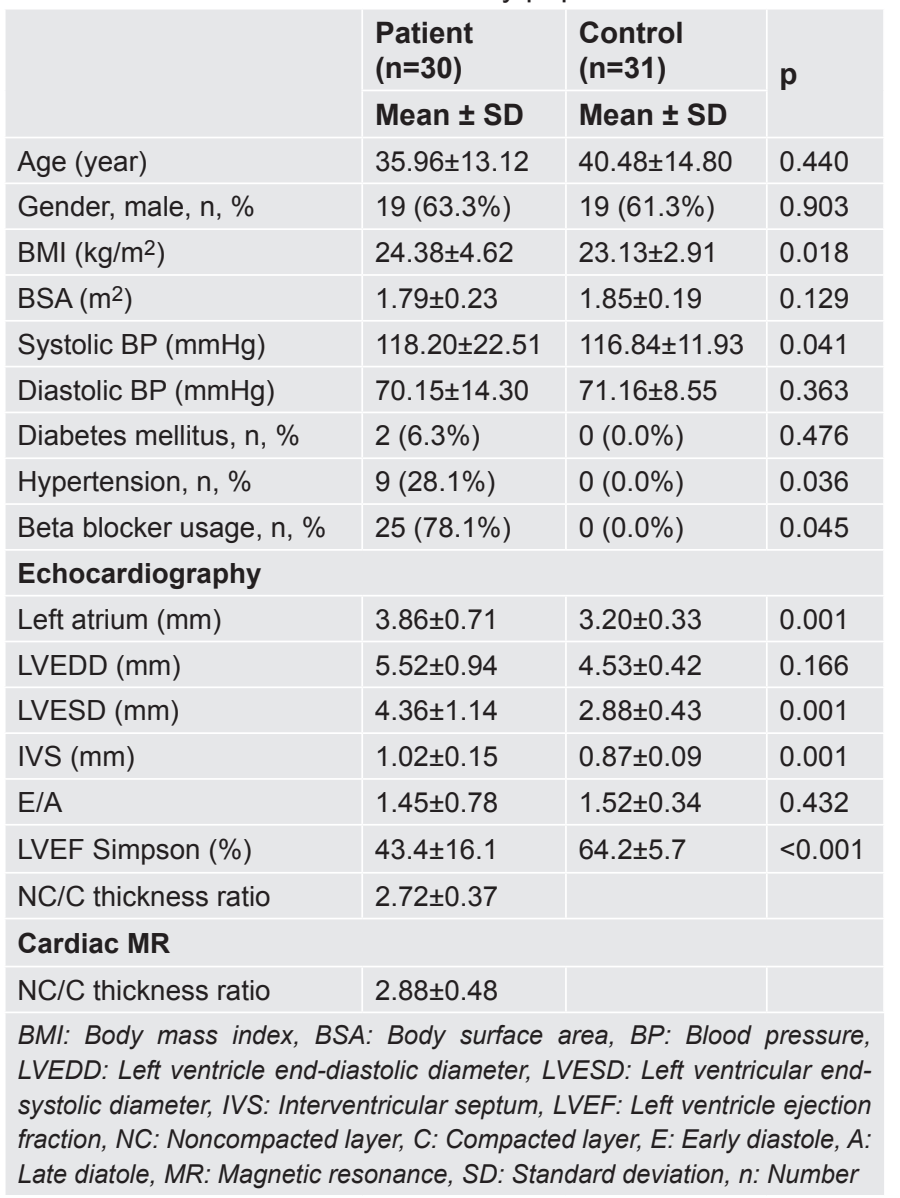

of arrhythmia, primarily parameters such as fQRS and QT duration were investigated and their relationship with arrhythmia and prognosis was determined ${ }^{(10,23)}$. Besides, the search for new electrocardiographic parameters concerning risk analysis in NCCM continues in recent years like T-wave amplitudes during ventricular repolarization in lead aVR (TaVR) and spatial QRS-T angle ${ }^{(11,24)}$.

In NCCM, heart rate changes can be monitored also with the effect of accompanying pathologies and the antiarrhythmic drug use. Bugan and Cekirdekci(25) investigated the cardiac autonomic features of NCCM and possible effects on mortality and achieved abnormal results in heart rate variability and heart rate turbulence.

QTd shows heterogeneity in ventricular repolarization secondary to changes in action potential duration and activation time. In a meta-analysis conducted by Bazoukis et al. ${ }^{(26)}$, as a result of the analysis of heart failure and coronary artery patients, QTd value was shown to have prognostic significance concerning arrhythmic events.

Table 2. Electrocardiographic parameters

\begin{tabular}{|l|l|l|l|}
\hline & $\begin{array}{l}\text { Patient } \\
(\mathbf{n = 3 0 )}\end{array}$ & $\begin{array}{l}\text { Control } \\
(\mathbf{n = 3 1 )}\end{array}$ & $\mathbf{p}$ \\
\hline Mean \pm SD & Mean \pm SD & \\
\hline Heart rate (/min) & $74.2 \pm 14.28$ & $74.65 \pm 10.32$ & 0.989 \\
\hline QRS (ms) & $95.73 \pm 13.83$ & $86.06 \pm 10.19$ & 0.006 \\
\hline QT $_{\text {max }}$ (ms) & $387.20 \pm 36.27$ & $371.29 \pm 25.23$ & 0.123 \\
\hline cQT $_{\text {max }}$ (ms) & $426.70 \pm 34.21$ & $412.68 \pm 33.01$ & 0.161 \\
\hline QT $_{\text {min }}$ (ms) & $365.33 \pm 37.71$ & $351.81 \pm 24.67$ & 0.233 \\
\hline cQT $_{\text {min }}$ (ms) & $402.46 \pm 35.04$ & $391.05 \pm 32.45$ & 0.290 \\
\hline QTd (ms) & $21.87 \pm 8.61$ & $19.48 \pm 5.07$ & 0.324 \\
\hline cQTd (ms) & $23.24 \pm 9.52$ & $21.62 \pm 5.69$ & 0.670 \\
\hline QT/QRS (iCEB) & $4.11 \pm 0.60$ & $4.37 \pm 0.61$ & 0.210 \\
\hline cQT/QRS (iCEBc) & $4.53 \pm 0.63$ & $4.84 \pm 0.60$ & 0.099 \\
\hline TPe (ms) & $79.93 \pm 9.04$ & $69.32 \pm 8.32$ & $<0.001$ \\
\hline cTPe (ms) & $88.27 \pm 10.90$ & $77.32 \pm 11.74$ & 0.001 \\
\hline TPe/QT & $0.207 \pm 0.026$ & $0.188 \pm 0.026$ & 0.008 \\
\hline TPe/cQT & $0.188 \pm 0.026$ & $0.168 \pm 0.022$ & 0.003 \\
\hline cTPe/cQT & $0.201 \pm 0.024$ & $0.181 \pm 0.024$ & 0.004 \\
\hline CQT & & & \\
\hline
\end{tabular}

$c Q T_{\text {max }}$ : Corrected $Q T_{\max }, c Q T_{\min }$ : Corrected $Q T_{\min }, Q T d: Q T$ dispersion, cQTd:corrected $Q T$ dispersion, for $Q T / Q R S$ and $T P E / Q T$ ratios $Q T_{\max }$ values were used, for $c Q T / Q R S$ and $T P e / c Q T$ ratios $c Q T_{\max }$ values were used, SD: Standard deviation, $n$ : Number 
It was also noted that this value was relatively high in patients with sudden cardiac death (SCD). In our study, QTd and cQTd values were relatively high compared to the controls $(p>0.05)$. In the study conducted by $\mathrm{Lu}$ et al. ${ }^{(19)}$ on the iCEB, which is a parameter related to the balance of ventricular depolarization and repolarization, the predictive properties of this new parameter were demonstrated for drug-related cardiac arrhythmias. In our study, there were no significant differences in iCEB and iCEBc values in NCCM patients compared to the control group.

TPe shows transmural dispersion of ventricular repolarization more accurately compared to the QT interval. In a study conducted by Chua et al. ${ }^{(18)}$, which investigated SCA patients, it has been shown that the cTPe value obtained after the correction of the TPe value according to heart rate using the Bazett or Fridericia formulas is predictive in terms of $\mathrm{SCD}$, and this value over $90 \mathrm{~ms}$ (with Bazett formula) increases this risk three times. In our study, the mean value of cTPe was $88.27 \pm 10.90$ and significantly higher than in the control group $(\mathrm{p}=0.001)$, and similarly TPe values were significantly higher $(\mathrm{p}<0.001)$. TPe/QT is another ECG parameter that can give an idea about transmural dispersion of left ventricular repolarization to be useful in predicting malignant cardiac arrhythmias in various diseases such as slow coronary flow, acute myocarditis, frequent outflow tract premature ventricular complexes ${ }^{(16,17,27)}$. In our study, TPe/QT, TPe/ cQT, and cTPe/cQT values were significantly higher than in the controls (all $\mathrm{p}$ values $<0.05$ ).

This is the first study to search the electrocardiographic features related to ventricular depolarization and repolarization in terms of QTd, $\mathrm{iCEB}$, TPe and TPe related parameters. In our study, we aimed to show the relationship (if any) between NCCM and the electrocardiographic parameters related to ventricular depolarization/repolarization, which are QTd, TPe, TPe/ QT ratio and iCEB. We showed a significant relationship with NCCM and TPe, cTPe, TPe/QT, TPe/cQT and cTPe/ cQT.

\section{Study Limitations}

In our study, we were able to evaluate a limited number of patients, also with the effect of NCCM which is a rare disease. Patients had some comorbidities like HT and DM. Because they are included in the optimal treatment protocol, some of the patients were using beta-blockers. Beta-blocker usage and comorbidities may have affected the results related to the electrocardiographic parameters.

\section{Conclusion}

We found a significant relationship between NCCM and TPe, cTPe, TPe/QT, TPe/cQT, cTPe/cQT parameters. We also observed that the values of QTd and cQTd were higher in NCCM compared to the control group. It can be thought that our study contributed to the literature with the present findings. The parameters which we investigated in our study and their relationship with arrhythmias in NCCM can be researched in further studies. Also, there is a need for further studies on the subject, with a larger number of patients. Thus, subgroup analysis can also be performed according to heart failure classification.

\section{Ethics}

Ethics Committee Approval: This study was approved by Ege University Ethics Committee, (date: 8/8/17, number: 17-6/12).

Informed Consent: Informed consent was obtained from all patients.

Peer-review: Externally peer-reviewed.

\section{Authorship Contributions}

Surgical and Medical Practices: O.A., İ.A., Concept: O.A., İ.A., Design: O.A., Data Collection or Processing: O.A., Analysis or Interpretation: O.A., İ.A., Literature Search: O.A., İ.A., Writing: O.A., İ.A.

Conflict of Interest: The authors declare no conflict of interest. 
Financial Disclosure: The authors declare no financial support by any grant or research sponsor, and no competing financial interest.

\section{References}

1. Chin TK, Perloff JK, Williams RG, et al. Isolated noncompaction of left ventricular myocardium. A study of eight cases. Circulation 1990;82:50713.

2. Pignatelli RH, McMahon CJ, Dreyer WJ, et al. Clinical characterization of left ventricular noncompaction in children: A relatively common form of cardiomyopathy. Circulation 2003;108:2672-8.

3. Ichida F, Hamamichi Y, Miyawaki T, et al. Clinical features of isolated noncompaction of the ventricular myocardium: long-term clinical course, hemodynamic properties, and genetic background. J Am Coll Cardiol 1999;34:233-40.

4. Hershberger RE, Lindenfeld J, Mestroni L, et al. Genetic evaluation of cardiomyopathy - a Heart Failure Society of America practice guideline. J Card Fail 2009;15:83-97.

5. Oechslin EN, Jost CHA, Rojas JR, et al. Long-term follow-up of 34 adults with isolated left ventricular noncompaction: a distinct cardiomyopathy with poor prognosis. J Am Coll Cardiol 2000;36:493-500.

6. Murphy RT, Thaman R, Blanes JG, et al. Natural history and familial characteristics of isolated left ventricular noncompaction. Eur Heart J 2005;26:187-92.

7. Steffel J, Kobza R, Oechslin E, et al. Electrocardiographic characteristics at initial diagnosis in patients with isolated left ventricular noncompaction. Am J Cardiol 2009;104:984-9.

8. Fiala M, Januska J, Bulkova V, et al. Septal ventricular tachycardia with alternating LBBB-RBBB morphology in isolated ventricular noncompaction. J Cardiovasc Electrophysiol 2010;21:704-7.

9. Kobza R, Steffel J, Erne P, et al. Implantable cardioverter-defi brillator and cardiac resynchronization therapy in patients with left ventricular noncompaction. Heart Rhythm 2010;7:1545-9.

10. Ning XH, Tang M, Chen KP, et al. The Prognostic Significance of Fragmented QRS in Patients With Left Ventricular Noncompaction Cardiomyopathy. Can J Cardiol 2012;28:508-14.

11. Cortez D, Sharma N, Imundo JR. Pediatric noncompaction patients with high spatial QRS-T angles are at increased risk for ventricular tachycardia. Ann Noninvasive Electrocardiol 2019;24:e12588.

12. Yan GX, Antzelevitch C. Cellular basis for the Brugada syndrome and other mechanisms of arrhythmogenesis associated with ST-segment elevation. Circulation 1999;100:1660-6.

13. Jacobson I, Carlsson L, Duke G. Beat by Beat QT interval variability, but not QT prolongation per se, predicts drug-induced torsades de pointes in the anaesthetized methoxamine-sensitized rabbit. J Pharmacol Toxicol Methods 2011;63:40-6.
14. Watanabe H, Knollmann BC. Mechanism underlying catecholaminergic polymorphic ventricular tachycardia and approaches to therapy. J Electrocardiol 2011;44:650-6

15. Gupta $P$, Patel $\mathrm{C}$, Patel $\mathrm{H}$, et al. $\mathrm{T}(\mathrm{p}-\mathrm{e}) / \mathrm{QT}$ ratio as an index of arrhythmogenesis. J Electrocardiol 2008;41:567-74.

16. Zehir R, Karabay CY, Kalaycı A. Evaluation of Tpe interval and Tpe/QT ratio in patients with slow coronary flow. Anatol J Cardiol 2015;15:463-7

17. Ucar FM, Ozturk C, Y1lmaztepe MA. Evaluation of Tp-e interval, Tp-e/ QT ratio and Tp-e/QTc ratio in patients with acute myocarditis. BMC Cardiovasc Disord 2019;19:232

18. Chua KC, Rusinaru C, Reinier K. Tpeak-to-Tend interval corrected for heart rate: A more precise measure of increased sudden death risk? Heart Rhythm 2016;13:2181-5

19. Lu HR, Yan GX, Gallacher DJ. A new biomarker - index of Cardiac Electrophysiological Balance (iCEB) - plays an important role in druginduced cardiac arrhythmias: beyond QT-prolongation and Torsades de Pointes (TdPs). J Pharmacol Toxicol Methods 2013;68:250-9.

20. Petersen SE, Selvanayagam JB, Wiesmann F, et al. Left ventricular noncompaction: insights from cardiovascular magnetic resonance imaging. $\mathrm{J}$ Am Coll Cardiol 2005;46:101-5.

21. Lang RM, Badano LP, Mor-Avi V, et al. Recommendations for cardiacchamber quantification by echocardiography in adults: an update from the American Society of Echocardiography and the European Association of Cardiovascular Imaging. J Am Soc Echocardiogr 2015;28:1 39.

22. Oechslin E, Jenni R. Left ventricular noncompaction from physiologic remodeling to noncompaction cardiomyopathy. J Am Coll Cardiol 2018;71:723-6.

23. Cetin MS, Ozcan Cetin EH, Canpolat U, et al. Usefulness of Fragmented QRS Complex to Predict Arrhythmic Events and Cardiovascular Mortality in Patients With Noncompaction Cardiomyopathy. Am J Cardiol 2016;117:1516-23

24. Ekizler FA, Cay S, Ulvan N, et al. Importance of lead aVR on predicting adverse cardiac events in patients with noncompaction cardiomyopathy. Ann Noninvasive Electrocardiol 2020;25:e12719.

25. Bugan B, Cekirdekci EI. Prognostic Significance of Heart Rate Turbulence Parameters in Patients with Noncompaction Cardiomyopathy. Cardiology 2019;142:56-62

26. Bazoukis G, Yeung C, Ho RWH, et al. Association of QT dispersion with mortality and arrhythmic events-A meta-analysis of observational studies. J Arrthythm 2019;11;36:105-15.

27. Kup A, Uslu A, Demir S, et al. Tp-Te interval and Tp-Te/QT ratio may be predictive of idiopathic ventricular tachycardia in patients with frequent outflow tract premature ventricular complexes Acta Cardiol 2020;14:1-6. 